\title{
Proceso logístico productivo de un centro de sangre regional: modelamiento y análisis
}

'Facultad de Ingeniería, Universidad del Desarrollo, Concepción, Chile.

${ }^{2}$ Centro de Sangre de Concepción, Chile. angeniero Civil Industrial, Ph.D. en Ingeniería Industrial.

bIngeniero Civil Industrial.

Recibido el 27 de octubre de 2010, aceptado el 7 de julio 2011.

Correspondencia a: Felipe Baesler, Ph.D. Facultad de Ingeniería Universidad del Desarrollo, Ainavillo 456, Concepción Chile.

Teléfono: 56-41-2268615,

Fax: 56-41-2268748. E-mail: fbaesler@ ingenieros.udd.cl

\author{
FELIPE BAESLER ${ }^{1, a}$, CRISTINA MARTÍNEZ², \\ EDUARDO YAKSIC ${ }^{1, \mathrm{~b}}$, CLAUDIA HERRERA ${ }^{2}$
}

\section{Logistic and production process in a regional blood center: modeling and analysis}

Background: The blood supply chain is a complex system that considers different interconnected elements that have to be synchronized correctly to satisfy in quality and quantity the final patient requirements. Aim: To determine the blood center maximum production capacity, as well as the determination of the necessary changes for a future production capacity expansion. Material and Methods: This work was developed in the Blood Center of Concepción, Chile, operations management tools were applied to model it and to propose improvement alternatives for the production process. The use of simulation is highlighted, which permitted the replication of the center behavior and the evaluation of expansion alternatives. Results: It is possible to absorb a $100 \%$ increment in blood demand, without making major changes or investments in the production process. Also it was possible to determine the subsequent steps in terms of investments in equipment and human resources for a future expansion of the center coverage. Conclusions: The techniques used to model the production process of the blood center of Concepcion, Chile, allowed us to analyze how it operates, to detect "bottle necks", and to support the decision making process for a future expansion of its capacity.

(Rev Med Chile 2011; 139: 1150-1156).

Key words: Computer simulation; Financial management, Hospital; Logistic models.
E 1 flujo de la sangre y de sus componentes derivados visto desde el donante hasta el paciente pueden ser considerados como en una cadena logística tradicional. Se recolecta la sangre desde los donantes, se separa en sus componentes en un centro y se envía estos productos a hospitales donde son transfundidos a los pacientes. La sincronización de cada uno de estos subprocesos resulta vital, si consideramos que los componentes sanguíneos son perecibles, destacando el caso de las plaquetas que alcanzan, en condiciones vitales seguras, los 5 días de vida. Igualmente desde un punto de vista productivo, la sincronización permite disminuir los cuellos de botella, reducir tiempos de espera, inventarios y mejorar la utilización de recursos. Por esto es imprescindible utilizar metodologías de análisis de procesos para obtener resultados que reduzcan costos de operación y mejoren la calidad del servicio otorgado al paciente, cumpliendo con las demandas en forma oportuna, segura y eficiente.

La literatura muestra algunos casos exitosos de utilización de herramientas de gestión de operaciones en centros de sangre. En el trabajo de Van Dijk et $\mathrm{al}^{1}$, se proponen modelos de inventarios a partir de enfoques de programación matemática. Este trabajo fue aplicado a un centro de sangre holandés, logrando disminuir las pérdidas de pla- 
quetas, de $20 \%$, a $1 \%$. Otro estudio similar publicado por Fontaine et $\mathrm{al}^{2}$, el cual fue realizado en el centro de sangre de Stanford (SBC), los resultados indican que es posible disminuir en 50\% las pérdidas de componentes sanguíneos si se implementan herramientas de cadena de suministro. En el caso particular de simulación de centros de sangre podemos destacar el trabajo de Rabinowitz ${ }^{3}$, donde se evalúan políticas de inventarios en el Banco de Sangre de Irving Geist. Se simularon tres escenarios para definir el nivel óptimo de inventario. Otro estudio corresponde a Cohen y Pierskalla ${ }^{4}$, quienes utilizaron simulación y optimización con el fin de minimizar la escasez y disminuir las pérdidas por vencimiento. Igualmente, Rytilä y Spens ${ }^{5}$ utilizan simulación en el Helsinki Blood Transfusion Center, para estudiar la producción de componentes sanguíneos. Los resultados del estudio permitieron reducir costos y aumentar la disponibilidad de sangre. Resultados similares fueron obtenidos por Navonil ${ }^{6}$ en el Servicio Nacional de Sangre de Gran Bretaña (NBS). Otro estudio más reciente corresponde a Haijema et $\mathrm{al}^{7}$, donde se modela un centro de sangre regional en Holanda. Los resultados definieron políticas de manejo de inventarios que permitieron disminuir significativamente las pérdidas por obsolescencia.

\section{Materiales y Métodos}

El año 2000 se creó el Centro de Sangre de Concepción para servir como modelo piloto y apoyar el desarrollo de la política de centralización de bancos de sangre, que a partir de la donación altruista, permita optimizar el uso de los recursos y mejorar la entrega de componentes sanguíneos a los hospitales. El centro actualmente concentra la producción de toda la región del Bío-Bío, cuya evolución histórica se muestran en la Tabla 1.

Estas donaciones son procesadas en el centro

Tabla 1. Donaciones históricas centro de sangre

\begin{tabular}{|cc|}
\hline Año & Donaciones \\
\hline 2004 & 17.649 \\
\hline 2005 & 21.238 \\
\hline 2006 & 23.337 \\
\hline 2007 & 28.309 \\
\hline 2008 & 35.108 \\
\hline
\end{tabular}

y transformadas principalmente en tres componentes, glóbulos rojos, plasma y plaquetas, además de crioprecipitados, los cuales se muestran en la Tabla 2.

El proceso logístico-productivo de la sangre está compuesto por una secuencia de estaciones interconectadas por flujos de datos y producto. La Figura 1 muestra el diagrama del proceso productivo del Centro de Sangre de Concepción.

La primera etapa contempla la donación, actualmente el Centro de Sangre de Concepción cuenta con cuatro sitios fijos de donaciones: Hospital Higueras de Talcahuano, Hospital Víctor Ríos Ruiz de Los Ángeles, Hospital Herminda Martin de Chillán, y la Casa del Donante de Concepción, además de las colectas móviles.

Esta primera etapa consiste en la extracción de la sangre, donde se realiza un examen de pre-donación para luego proceder a la extracción donde se obtiene una bolsa de $450 \mathrm{ml}$ de producto que será procesado en las siguientes etapas, además de dos muestras para análisis posteriores. Al llegar el producto al centro, se ingresan los datos al sistema informático y se pesan las bolsas.

La bolsa principal es ubicada dentro de un recipiente llamado liner para su posterior centrifugación, proceso que definiremos como "Preparación Centrifugación", luego se procede con el "Centrifugado", logrando la separación de componentes según sus pesos y densidades. Luego se procede al "Fraccionamiento", donde un equipo separa los componentes en una bolsa distinta, quedando los glóbulos rojos en una bolsa, el plasma en otra y dejando en la bolsa original el buffy-coat, compuesto de restos de glóbulos rojos, plasma, glóbulos blancos y plaquetas, siendo este último componente el producto final de una nueva separación.

Luego, cada componente sigue caminos distintos, el buffy-coat queda almacenado en reposo para

Tabla 2. Producción histórica centro de sangre

\begin{tabular}{|cccc|}
\hline Año & Glóbulos rojos & Plasma & Plaquetas \\
\hline 2004 & 17.128 & 11.780 & 13.772 \\
\hline 2005 & 20.659 & 12.991 & 17.622 \\
\hline 2006 & 22.702 & 16.776 & 18.264 \\
\hline 2007 & 25.433 & 20.965 & 23.042 \\
\hline 2008 & 33.872 & 24.243 & 28.914 \\
\hline
\end{tabular}




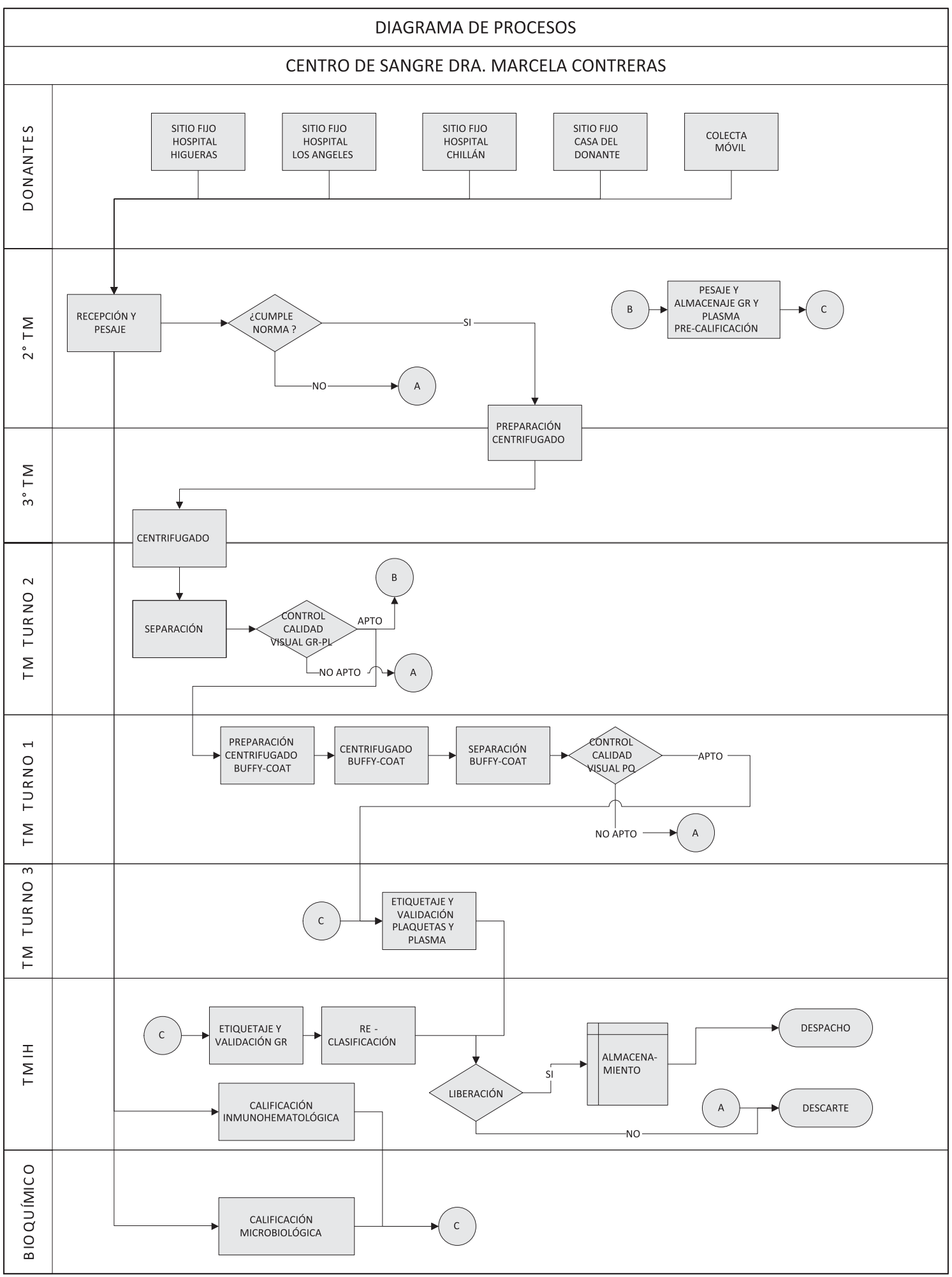

Figura 1. Diagrama de procesos centro de sangre de Concepción. 
su re-procesamiento al día siguiente, los glóbulos rojos son pesados para verificar que cumplan con la norma, luego se ingresan los datos al sistema informático y se refrigeran a la espera de los estudios microbiológicos e inmunohematológicos que permitirán el etiquetado. El plasma fresco también es pesado, luego sufre un congelado rápido y posteriormente es almacenado a -30 grados Celsius.

Secuencialmente el proceso continúa con la elaboración de plaquetas a partir de buffy-coat, para ello son centrifugadas y luego separadas. Al día siguiente se procede a realizar los análisis microbiológicos e inmunohematológicos, para luego etiquetar los productos que pasen los análisis.

Como una etapa final se realiza una re-clasificación manual, $\mathrm{ABO}$ y $\mathrm{Rh}$, en la cual se verifica nuevamente el grupo de sangre en la bolsa y se chequea que sea el mismo impreso en la etiqueta. El almacenaje se realiza en condiciones distintas dependiendo del producto, glóbulos rojos, plasma o plaquetas. Finalmente, los componentes sanguíneos serán distribuidos.

Cabe mencionar que la etapa de microbiología e inmunohematología se modeló en forma agregada como un macroproceso sin considerar las subactividades y procedimientos realizados internamente.

Este proceso fue modelado utilizando simulación de procesos. Esta metodología permite emular computacionalmente el comportamiento del proceso real presentado en la Figura 1, y así estudiar el desempeño que tendrá el proceso real cuando se someta a cambios futuros, como variaciones en la demanda, y modificaciones en la disponibilidad de recurso. Otra ventaja es la posibilidad de incorporar la aleatoriedad propia de las actividades del proceso, a través de la determinación de distribuciones estadísticas. Mayores detalles relacionados con la técnica de simulación, en referencias 8 y 9. Para el desarrollo del modelo de simulación fue necesario seguir el siguiente procedimiento:

a. Definición del problema: Se definen claramente los objetivos y alcances del proyecto de simulación.

b. Recolección y análisis de datos: Se recolecta la información relativa a tiempos de procesos, volúmenes de donaciones, etc., y se realiza un análisis estadístico para la confección de distribuciones de probabilidad para carac- terizar el comportamiento de cada actividad involucrada en el proceso. Este modelo consta de 20 actividades como, pesaje de la sangre, separación de la sangre, etiquetaje de glóbulos, etc., además del número de donaciones diarias de cada sitio de donación y los despachos a cada unidad transfusional. En total se recolectaron datos para 31 actividades y se aplicaron test de bondad de ajuste para ajustar una distribución estadística a cada actividad. A modo de ejemplo, el tiempo de duración de la actividad "etiquetaje de glóbulos" pudo ser ajustada a una distribución Gamma con parámetros alfa 1.36, beta 1.18 y una constante igual a 12 segundos.

c. Desarrollo del modelo conceptual: Esta actividad considera el levantamiento del proceso, diagramación de actividades y determinación de las interrelaciones entre cada sub-actividad del proceso.

d. Construcción del modelo computacional: El modelo es creado en un software especializado a través de la incorporación de código computacional que representa la interconexión entre las distintas actividades del proceso mostrado en la Figura 1, al igual que su tiempo de duración expresado a través de las distribuciones estadísticas obtenidas en el punto anterior.

e. Verificación y Validación: Se verifica y valida el funcionamiento del modelo con el fin de determinar si su desempeño es una representación del comportamiento del proceso real. Esto se hace comparando los resultados obtenidos del modelo computacional con datos reales del proceso y determinado la inexistencia de diferencia significativa entre ambos.

f. Experimentación y análisis de resultados: Finalmente, se simula el proceso para que replique el comportamiento del centro de sangre durante un tiempo determinado. El modelo es capaz de generar eventos que representan las llegadas de donaciones, tiempos de cada actividad, despachos, etc. De esta manera es posible evaluar el comportamiento del Centro por un tiempo prolongado en forma acelerada a través del modelo. Se realizan experimentos modificando condiciones operacionales del sistema y se analiza su comportamiento sin necesidad de implementar esos cambios en el proceso real. 
Resultados

Utilizando el procedimiento presentado en la sección anterior, se representó el comportamiento del sistema mediante un modelo construido con el software de simulación ARENA 12.0. El objetivo principal de este análisis se centró en la determinación de la capacidad máxima del centro de sangre. Este objetivo es de suma importancia debido a la nueva política pública que apunta a la centralización de los Centros de Sangre en Chile, la cual define sólo tres centros productores para todo el país. En este contexto el Centro debería concentrar la producción de una extensión significativa del sur del país, por lo cual en el mediano plazo deberá ser capaz de aumentar su capacidad productiva en forma paulatina en prácticamente tres veces.

Cabe mencionar que este estudio no se abocó a evaluar alternativas para la disminución de costos operativos, optimización de inventarios o minimizar las pérdidas por obsolescencia. Sin embargo, puede ser adaptado para lograr dichos objetivos.

Para este análisis se realizaron 9 experimentos distintos de simulación, donde cada uno considera $20 \%$ de aumento de las donaciones de sitio fijo. Como resultado, el modelo arroja indicadores relacionados a tiempo de espera y utilización de recursos, que finalmente permiten determinar los cuellos de botella del sistema y por ende concluir en relación a la capacidad máxima del sistema.
Realizados estos escenarios, se puso énfasis en la utilización de los recursos separadores, centrífugas y tecnólogo médico de turno 2, y los tiempos de espera en las colas. Los resultados se muestran en las Tablas 3 y 4.

La Tabla 3 muestra que la utilización del recurso separador es significativamente más alta que el resto de los recursos, evidenciando que el cuello de botella del sistema se encuentra en esta etapa. Se ve la evolución de la utilización de los recursos a medida que se aumentan las donaciones, destacando la utilización de los separadores que en el escenario de $100 \%$, alcanza $90,65 \%$ de utilización. Los escenarios siguientes llegan al 100\% de utilización de los separadores, lo cual indica que la capacidad máxima del sistema se ve restringida por este recurso cuello de botella cuando se aumentan las donaciones en $100 \%$. El resto de los recursos se aprecian con bastante capacidad disponible. La Tabla 4 presenta los tiempos de espera promedio de las unidades antes de los procesos de centrifugación y separación. Nuevamente se puede concluir que el cuello de botella del proceso es la estación Separación ya que sus tiempos de espera superan significativamente al resto de los recursos.

Bajo estos criterios, se considera que la capacidad máxima funcional del centro sin realizar cambios ni caer en sobre tiempos de personal es de $100 \%$. Es decir, el doble de las donaciones actuales (224 diarias) sin necesidad de aumentar la disponibilidad de ninguno de los recursos existentes.

Tabla 3. Análisis de sensibilidad utilización de recursos

\begin{tabular}{|lcccccccc|}
\hline \multicolumn{7}{|l}{ Utilización de recursos (\%) } & para cada escenario \\
\hline Escenario & $20 \%$ & $40 \%$ & $60 \%$ & $80 \%$ & $100 \%$ & $120 \%$ & $140 \%$ & $160 \%$ \\
\hline Separadores & 65,16 & 70,29 & 79,68 & 85,86 & 90,65 & 100,00 & 100,00 & 100,00 \\
\hline Centrífugas & 13,58 & 15,30 & 17,20 & 18,86 & 20,67 & 22,43 & 24,16 & 25,83 \\
\hline TM Turno 2 & 36,87 & 41,74 & 46,98 & 51,59 & 56,20 & 60,59 & 64,33 & 67,68 \\
\hline
\end{tabular}

Tabla 4. Análisis de sensibilidad tiempos de espera

\begin{tabular}{|c|c|c|c|c|c|c|c|c|}
\hline \multicolumn{9}{|c|}{ Tiempo de espera para cada escenario (minutos) } \\
\hline Escenario & $20 \%$ & $40 \%$ & $60 \%$ & $80 \%$ & $100 \%$ & $120 \%$ & $140 \%$ & $160 \%$ \\
\hline Centrifugación & 3,33 & 3,90 & 4,55 & 5,22 & 5,98 & 6,68 & 7,43 & 8,22 \\
\hline Separación & 17,36 & 21,15 & 28,01 & 36,52 & 79,20 & 117,90 & 177,20 & 236,49 \\
\hline
\end{tabular}




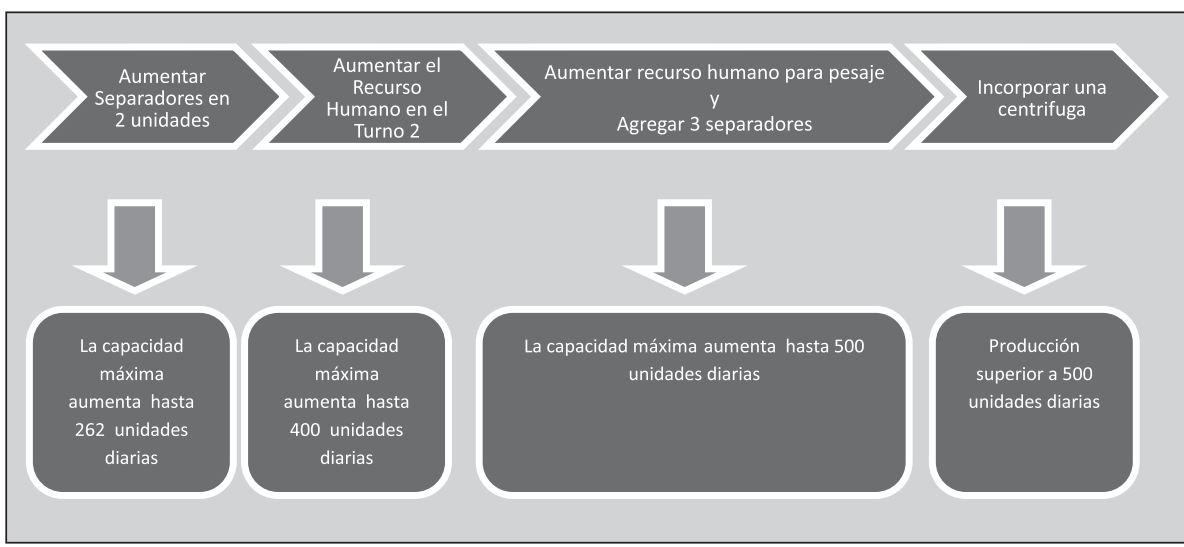

Figura 2. Esquema de Inversiones.

\section{Aumento de la demanda en el largo plazo}

Un segundo objetivo del estudio se centró en analizar una expansión de la capacidad de producción superior las 224 unidades diarias. Un volumen de esta dimensión requeriría inversiones o incrementos de recursos que deben ser determinados en función a los cambios que los cuellos de botellas sufrirán a medida que se incremente el volumen de producción. Este análisis permite determinar en qué estación se producen los cuellos de botella y qué tipo de inversiones son necesarias para absorber dichos niveles de producción. Los resultados de estas nuevas simulaciones se muestran a continuación.

Con los horarios del modelo original, aumentando la demanda en forma discreta para cada experimento, se concluye lo siguiente.

- Al aumentar a 243 unidades, es necesario incorporar una nueva máquina separadora.

- Para 262 unidades, 8 separadores es suficiente, pero el operario de la tarde no alcanza a montar las bolsas en las máquinas. Se necesita un operario de cooperación en dichas operaciones.

- Hasta 400 unidades pueden ser procesado con 8 separadores y 2 operarios de turno tarde.

- Sobre esta cantidad el cuello de botella se traspasa al pesaje. Por esta razón, cualquier aumento sobre esta cantidad requiere la incorporación de un operario para el pesaje.

- Si se agregan 3 separadores adicionales es posible procesar hasta 500 unidades diarias.

- Sobre 500 unidades el cuello de botella se traspasa a las centrífugas por lo cual se debería incorporar una adicional.

El camino a seguir por el Centro de Sangre de
Concepción para su crecimiento se muestra en la Figura 2.

\section{Discusión}

En este trabajo se ha mostrado el beneficio de utilizar metodologías del ámbito de la gestión de operaciones, para el análisis de procesos de centros de sangre. El modelo de simulación permitió identificar los cuellos de botella que restringen la capacidad de producción del sistema. Primero se determina la capacidad actual del proceso de producción, llegando a concluir que con las condiciones, equipamiento y dotación de personal existente es posible duplicar la producción actual. Segundo, se realizó un análisis de expansión de capacidad, determinando los cambios necesarios para absorber futuros aumentos de demanda. La importancia de este análisis radica en la información que aporta a la toma de decisiones futuras del centro de sangre, ya que espera para el mediano plazo una demanda de 369 unidades diarias de sangre, como resultado del programa de centralización de los Centros de Sangre en Chile. Esta cantidad implica un aumento de casi $200 \%$ en relación a la capacidad actual, obligando a la Dirección del Centro a rediseñar el proceso para triplicar su capacidad en el mediano plazo, situación sumamente compleja, que requiere un proceso de toma de decisiones orientadas a programar nuevas contrataciones e inversiones en equipamiento. Es interesante la conclusión que la incorporación de una centrífuga no es relevante hasta que la demanda supere las 500 unidades dia- 
rias, permitiendo así, priorizar las inversiones en cada etapa de expansión. Cabe mencionar que este plan de inversiones no considera las necesidades de equipamiento de respaldo, orientado a mantener el funcionamiento continuo del proceso frente a fallas de equipos. Por esta razón los resultados entregados deben ser interpretados en condiciones ideales de operación, e implementados en función de la evidencia histórica y la experiencia de los tomadores de decisiones.

Es importante comentar que la etapa de pesaje puede ser eliminada del proceso si se usa un separador automatizado, que pesa automáticamente y entrega la información al sistema informático.

Finalmente, se debe destacar que este estudio muestra una metodología para enfrentar el mejoramiento continuo de centros de sangre, el cual es totalmente replicable para su aplicación en otros centros e incluso a otros procesos del ámbito de la salud.

\section{Referencias}

1. Van Dijk N, Haijema R, Van der Wal J, Sibinga C. Blood platelet production: a novel approach for practical opti- mization. Transfusion 2009; 49 (3): 411-20.

2. Fontaine M, Chung Y, Rogers W, Sussmann, H, Quach P, Galel S, et al. Improving platelet supply chains through collaborations between blood centers and transfusion services. Transfusion 2009; 49 (10): 2040-7.

3. Rabinowitz M. Blood Bank Inventory Policies: A Computer Simulation. Health Services Research 1973: 27182.

4. Cohen M, Pierskalla W. Simulation of Blood Bank System; Department of Decision Sciences. The Wharton School 1979.

5. Rytilä J, Spens K. Using simulation to increase efficiency in blood supply chains. Management Research News 2006; 29 (12): 801-18.

6. Navonil M. Distributed Simulation with COTS Simulation Packages: A case study in health care supply chain simulation 2006; Winter Simulation Conference: 113642.

7. Haijema R, Van der Wal J, Van Dijk N. Blood platelet production: Optimization by dynamic programming and simulation. Computers \& Operations Research 2007; 34 (3): 760-79.

8. Law A. Simulation Modeling and Analysis. McGraw-Hill, 2007.

9. Banks J, Nelson B, Carson J, Nicol D, Carson II J. Discrete-Event System Simulation. Prentice Hall, 2009. 